\title{
ANÁLISE UNIVARIADA E MULTIVARIADA DE CARACTERÍSTICAS REPRODUTIVAS E PRODUTIVAS DE CABRAS LEITEIRAS UTILIZANDO INFERÊNCIA BAYESIANA
}

\author{
SANTOS, Natanael Pereira da Silva ${ }^{1}$ \\ SARMENTO, José Lindenberg Rocha ${ }^{2}$ \\ PIMENTA FILHO, Edgard Cavalcanti ${ }^{3}$ \\ CAMPELO, José Elivalto Guimarães ${ }^{4}$ \\ FIGUEIREDO FILHO, Luiz Antonio Silva ${ }^{1}$ \\ SOUSA JÚNIOR, Severino Cavalcante $\mathrm{de}^{2}$ \\ REGO NETO, Aurino de Araújo
}

\begin{abstract}
RESUMO: Esta pesquisa objetivou estudar efeitos ambientais e genéticos sobre características reprodutivas e produtivas de cabras mestiças exploradas para a produção leiteira. Foram estimados componentes de (co)variância e parâmetros genéticos das características idade ao primeiro parto, intervalo de partos e produção de leite em análises unicaracterística e multicaracterística, utilizando-se a estatística Bayesiana sob modelo animal. As análises genéticas foram realizadas com cadeias de 1.000 .000 de ciclos. Considerou-se o burn-in dos 100.000 valores iniciais sendo tomados valores a cada 300 ciclos, para se obter a distribuição a posteriori com 3.000 amostras. As médias obtidas para idade ao primeiro parto, intervalo de partos e produção de leite foram 581,68 $\pm 79,44 ; 322,89 \pm 132,02$ dias e $226,99 \pm 89,72 \mathrm{~kg}$ de leite, respectivamente. Todos os efeitos fixos incluídos no modelo foram significativos, exceto o tipo de nascimento para o intervalo de partos e produção de leite. As herdabilidade em análise unicaracterística foram 0,14; 0,05 e 0,10 para idade ao primeiro parto, intervalo de partos e produção de leite, respectivamente. Na mesma ordem, em análise multicaracterística, as herdabilidades foram de 0,16; 0,06 e 0,11. As estimativas de repetibilidade para as características intervalo de partos e produção de leite foram 0,10 e 0,20 respectivamente. As características estudadas mostraram-se fortemente influenciadas pelo ambiente. As análises multicaracterísticas levaram a estimativas ligeiramente maiores de herdabilidade.
\end{abstract}

Palavras-chave: Caprinos. Componentes de variância, Inferência bayesiana. Parâmetros genéticos

\section{UNIVARIATE AND MULTIVARIATE ANALYSIS OF CHARACTERS REPRODUCTIVE AND PRODUCTIVE ON MILK GOATS USING BAYESIAN INFERENCE}

SUMMARY: This research aimed to study environmental and genetic effects on reproductive and productive traits of goats exploited for milk production. (Co)Variance components and genetic trait parameters for age at first calving, calving interval, and milk production in univariate and multivariate analysis, using Bayesian statistics under animal model, were estimated. Genetic analyses were performed with chains of 1,000,000 cycles. The burn-in of the 100,000 baseline values was considered and these ones were taken every 300 cycles to obtain a posteriori distribution with 3,000 samples. The averages obtained for age at first calving, calving interval, and milk production were 581.68 $\pm 79.44 ; 322.89 \pm 132.02$ days, and $226.99 \pm 89.72 \mathrm{~kg}$ of milk respectively. All the effects included in the model were significant, except the type of birth one for calving interval, and milk production. The univariate analyses showed heritability values around $0.14,0.05$, and 0.10 for age at first calving, calving interval, and milk production respectively. In the same order, in multivariate analysis, the heritabilities were $0.16,0.06$, and 0.11 . Repeatability estimates for the calving interval, and milk production traits were 0.10 and 0.20 respectively. The traits analyzes showed to be strongly influenced by the environment. The multivariate analysis led to slightly higher estimates of heritability.

Keywords: Goats, Variance components. Bayesian inference. Genetic parameter.

\footnotetext{
${ }^{1}$ Programa de Pós-Graduação em Ciência Animal da Universidade Federal do Piauí - UFPI/Teresina. E-mail: natanaelpss@ hotmail.com.

${ }^{2}$ Professor, Doutor do Campus Cinobelina Elvas - UFPI/Bom Jesus. Bolsista CNPq.

${ }^{3}$ Professor, Pós-Doutor Do Campus de Areia - UFPB/CCA - Paraíba.

${ }^{4}$ Professor, Doutor do Campus Petrônio Portela - UFPI/Teresina. (CCA / Departamento de Zootecnia).
} 


\section{INTRODUÇÃO}

O grau de eficiência do processo de seleção é observado pela mudança genética que ocorre nos rebanhos, tanto com base em uma ou em várias características de interesse. Com a avaliação genética é possível verificar se está havendo melhoramento genético no rebanho e se a taxa de ganho se apresenta satisfatória. Caso a resposta à seleção não seja favorável, é preciso identificar as falhas e estabelecer métodos mais eficientes, que certamente terão por base, parâmetros genéticos estimados com qualidade.

O estudo de características reprodutivas por meio de modelos multicaracterísticas, pode ser mais consistente quando comparado ao uso de modelos unicaracterística, pois considera as relações entre as características. Em análises multicaracterísticas, a avaliação do animal para uma característica se faz pela contribuição de todas as outras envolvidas na análise (LASSEN, 2007).

Do ponto de vista do melhoramento genético, as estimativas de parâmetros obtidas, utilizando-se métodos apropriados, são essenciais, pois estimativas acuradas com o menor erro possível são requeridas ao estabelecimento de eficientes programas de seleção. Assim, os Métodos de Monte Carlo via Cadeias de Markov (MCMC), dentre os quais se destaca a amostragem de Gibbs, podem ser utilizados como ferramenta que propicia a inferência Bayesiana, a partir da qual são obtidas as estimativas dos componentes de variância e parâmetros genéticos (FARIA et al., 2007).

A Inferência Bayesiana tem sido intensamente empregada na estimação de parâmetros genéticos em estudos que utilizam diferentes modelos, principalmente para características com poucos dados amostrais (CARNEIRO JÚNIOR et al., 2005; FALCÃO et al., 2009).

Esta pesquisa objetivou avaliar os aspectos ambientais e estimar os componentes de (co)variância e parâmetros genéticos de características reprodutivas (idade ao primeiro parto e intervalo de partos) e produtiva (produção de leite) em cabras leiteiras, utilizando Inferência Bayesiana, sob modelos uni e multicaracterística.

\section{MATERIAL E MÉTODOS}

Esta pesquisa foi realizada com dados do rebanho caprino da Fazenda Carnaúba, pertencente à Agropecuária Manoel Dantas Ltda. (AMDA), situada no município de Taperoá, microrregião do Cariri Ocidental da Paraíba. Os registros de idade ao primeiro parto (IPP), intervalo de partos (IP) e produção total de leite (PL) foram obtidos a partir do controle reprodutivo e produtivo das cabras mestiças oriundas do cruzamento de reprodutores da raça Pardo Alpino com fêmeas do tipo naturalizado Gurguéia, coletados com representatividade durante 8 (oito) anos consecutivos.

Os animais foram criados em sistema semi-intensivo e alimentados com capim-buffel (Cenchrus ciliaris L.) e vegetação nativa durante a estação chuvosa, e capim-elefante (Pennisetum purpureum schum.), palma forrageira (Opuntia sp.), raspa de mandioca (Manihot sativa), bagaço de cana hidrolisado 
(Sacharum oficcinarum) e concentrado protéico, na estação seca, além de sal mineral à vontade no aprisco.

A detecção do cio foi realizada visualmente pela manhã e à tarde. Havendo a identificação de cio, a cabra era levada à baia do reprodutor correspondente, onde ocorria a cobertura e depois retornava ao lote. A monta era do tipo natural controlada, sem estação de monta definida. Após a primeira parição, as cabras eram incorporadas no lote dos animais adultos.

Para gerar o arquivo utilizado nas análises partiu-se de um banco de dados com 1.939 registros onde foram impostas restrições. Assim, permaneceram no arquivo apenas, cabras com pai e mãe conhecidos, que apresentavam no máximo sete parições, bem como grupos de contemporâneos com no mínimo 3 animais, restando 532 registros para as análises (Tabela 1).

Após a edição dos dados, a partir das fichas de produção e reprodução do rebanho, editou-se um arquivo contendo o número da cabra, pai e mãe, data de nascimento, datas dos partos, datas das coberturas, pesos antes e durante a gestação, ordem e intervalo de partos, número de cabritos nascidos por parto, idade aos partos e produção de leite, bem como os grupos de contemporâneos.

Tabela 1. Estatística descritiva dos dados para as análises

\begin{tabular}{cccccccc}
\hline Característica & N & Média & DP & CV\% & Mínimo & Máximo & GC \\
\hline IPP (dias) & 392 & 581,68 & 79,44 & 13,66 & 378,00 & 890,00 & 16 \\
IP (dias) & 532 & 322,89 & 132,02 & 40,89 & 124,00 & 828,00 & 78 \\
PL (kg) & 324 & 226,99 & 89,72 & 39,53 & 90,00 & 563,00 & 69
\end{tabular}

IPP - idade ao primeiro parto; IP - intervalo de partos; PL - produção de leite; $\mathrm{n}$ - número de observações; DP desvio padrão; CV - coeficiente de variação; GC - número de grupos de contemporâneos.

Foram considerados grupos de contemporâneos (GC) distintos para as características. Os grupos de contemporâneos para característica IPP (GC1) contemplavam animais que pariram no mesmo ano (1 a 8) e mesma estação ( 1 e 2), onde a estação 1 corresponde ao período chuvoso na região, que vai de fevereiro a junho, e a estação 2, ao período seco, que vai de julho a janeiro do ano seguinte. Para as características IP e PL os grupos de contemporâneos contemplavam animais que pariram no mesmo ano (1 a 8), mesma estação (1 e 2), além da mesma ordem de parição (1 a 7) e do tipo de nascimento (1 para simples e 2 para múltiplo).

Para formação dos arquivos de dados e de pedigree, análise de consistência dos dados e a verificação dos efeitos ambientais capazes de influenciar as características IPP, IP e PL, foram utilizados os procedimentos MEANS e GLM do programa SAS (Statistical Analysis Systems, versão 9.2).

Para análise dos efeitos ambientais que podem influenciar as características, foram utilizados dois modelos estatísticos distintos. O modelo 1 foi utilizado para análise da IPP e o modelo 2 foi utilizado para IP e PL, como seguem: 


$$
\begin{aligned}
& \text { Modelo 1: } Y_{i j k l m}=\mu+A_{i}+E_{j}+N_{k}+G C 1_{l}+\varepsilon_{i j k l m}, \\
& \text { Modelo 2: } Y_{i j k l m n}=\mu+A_{i}+E_{j}+N_{k}+G C 2_{l}+O_{m}+\varepsilon_{i j k l m n}
\end{aligned}
$$

em que, $y$ é a resposta do animal de acordo com a característica avaliada (IPP, IP e PL); $\mu$ é a média geral da característica; $A_{i}$ é o efeito do ano de parição; $E_{j}$ é o efeito da estação de parição; $N_{k}$ é o efeito do tipo de nascimento; $G C_{l}$ é o efeito do grupo de contemporâneos (GC1 e GC2, de acordo com a característica estudada); $O_{m}$ é o efeito da ordem de parição; e $\varepsilon$ é o erro aleatório independente distribuído com média zero e variância $\sigma^{2}$.

Os componentes de (co)variância e parâmetros genéticos, para fins de comparação, mediante análises Bayesiana uni e multicaracterística, sob modelo animal, foram estimados por meio do aplicativo MTGSAM (Multiple Trait Gibbs Sampler for Animal Models) (VAN TASSELL; VAN VLECK, 1995). Na modelagem, considerou-se a idade do animal ao parto como covariável com efeito quadrático para as características IP e PL. Consideraram-se os grupos de contemporâneos como efeito fixo, de acordo com a solicitação do programa. Entretanto, na análise Bayesiana os efeitos fixos e aleatórios inclusos no modelo são considerados variáveis aleatórias.

Foram utilizados dois modelos diferentes nas análises. O primeiro não contendo o efeito de ambiente permanente para avaliação da característica IPP e segundo modelo contemplando este efeito para as características IP e PL. Dessa forma, os modelos animais em notação matricial podem ser representados assim:

$$
\begin{aligned}
& \text { Modelo 1: } y=X b+Z a+e, \\
& \text { Modelo 2: } y=X b+Z a+W c+e,
\end{aligned}
$$

em que $y$ é o vetor das observações da característica medida nos animais (IPP, IP e PL); $b$ é o vetor de efeitos "fixos"; $a$ é o vetor de efeitos aleatórios que representa os valores genéticos aditivos diretos de cada animal; $c$ é o vetor de efeitos de ambiente permanente (não correlacionado) para as características IP e PL; $e$ é o vetor de erros aleatórios; $X, Z$ e $W$ são matrizes de incidência que relacionam as observações aos efeitos fixos e aos efeitos aleatórios aditivo direto e de ambiente permanente, respectivamente.

Para os modelos adotados, sob o enfoque bayesiano, adotaram-se as seguintes pressuposições: em análise unicaracterística assume-se que a distribuição condicional dos dados $(y)$, dados, $b, a, c$ e $\sigma_{e}^{2}$ é normal e multivariada, assim:

$$
y\left|b, a, c, \sigma_{e}^{2} \sim N \varangle b+Z a+W c+I \sigma_{e}^{2} ; a\right| \sigma_{a}^{2} \sim N \boldsymbol{Q}, A \sigma_{a}^{2} ; c\left|\sigma_{c}^{2} \sim N \mathbf{Q}, I \sigma_{c}^{2} ; e\right| \sigma_{e}^{2} \sim N \mathbf{Q}, I \sigma_{e}^{2}, \mathrm{em}
$$

que $\sigma_{a}^{2}, \sigma_{c}^{2}$ e $\sigma_{e}^{2}$ são componentes de variância genético aditivo direto, de ambiente permanente e residual, respectivamente, $A$, é matriz de numeradores do coeficiente de parentesco de Wrigth e $I$ é matriz identidade de ordem igual ao número de animais com observações. Usualmente $b$ assume priori 
não informativa, pois dentro do contexto Bayesiano não há distinção entre efeitos fixos e aleatórios e dessa forma: $P \ll g$ constante.

Em análise multicaracterística o modelo completo em notação matricial é dado como segue:

$$
\left[\begin{array}{l}
y_{1} \\
y_{2}
\end{array}\right]=\left[\begin{array}{ll}
X_{1} & 0 \\
o & X_{2}
\end{array}\right]\left[\begin{array}{l}
b_{1} \\
b_{2}
\end{array}\right]+\left[\begin{array}{ll}
Z_{1} & 0 \\
0 & Z_{2}
\end{array}\right]\left[\begin{array}{l}
a_{1} \\
a_{2}
\end{array}\right]+\left[\begin{array}{ll}
W_{1} & 0 \\
0 & W_{2}
\end{array}\right]\left[\begin{array}{l}
c_{1} \\
c_{2}
\end{array}\right]+\left[\begin{array}{l}
e_{1} \\
e_{2}
\end{array}\right],
$$

Pressupõe-se que: $y \mid b, a, c, \sigma_{e}^{2} \sim N M V \otimes b+Z a+W c, R^{-}$, com $R=\operatorname{diag} \sigma_{e}^{2}$, sendo essa a função de verossimilhança.

As pressuposições acerca das distribuições a priori para os parâmetros desconhecidos são consideradas como a seguir:

$a \mid G_{0} \sim N M V \emptyset, G_{-}^{-}$com $G=A \otimes G_{0}$, em que $A$ é a matriz de parentesco entre os animais e $G_{0}$ é a matriz de (co)variância genética aditiva entre as características;

$c \mid C_{0} \sim N M V \mathbb{Q}, C_{-}^{-}$, com $C=I \otimes C_{0}$, em que I é uma matriz identidade e $C_{0}$ é a matriz de (co)variância de ambiente permanente;

$G_{0} \mid v_{a}, S_{a}^{2} \sim W^{-1} \mathbf{C}_{a}, v_{a}, S_{a}^{2} ;$

$C_{0} \mid v_{c}, S_{c}^{2} \sim W^{-1} \zeta_{c}, v_{c}, S_{c}^{2}$, em que $v_{a}, S_{a}^{2}$ e $v_{c}, S_{c}^{2}$ representam o grau de confiança e os valores $a$ priori para as (co) variâncias genética aditiva e de ambiente permanente, respectivamente;

$\sigma_{e}^{2} \mid v_{e}, S_{e}^{2} \sim \chi^{-2}\left(\mathbf{L}_{e} S_{e}^{2}\right]_{-}^{2}$ em que $v_{e}, S_{e}^{2}$ representam o grau de confiança e os valores a priori para do resíduo; $b \alpha$ constante; e $N M V, W^{-1}$ e $\chi^{-2}$ refere-se às distribuições normal multivariada, Wishart invertida e Qui-quadrado invertida, respectivamente e $\otimes=$ operador produto-direto.

O grau de liberdade correspondente à distribuição Wishart Invertida, que indica o grau de confiabilidade da distribuição inicial (্), foi nulo (flat) para todas as variâncias iniciais, ou seja, não refletiu grau de conhecimento sobre os parâmetros $\ell_{=0}^{-}$.

Foram realizadas análises com cadeias de 1 milhão de ciclos. Considerou-se o burn-in dos 100 mil valores iniciais. Para evitar a redundância das informações causada pelas correlações seriais entre os valores gerados subsequentemente, foram tomadas amostras a cada 300 ciclos (Intervalos de amostragem Thinning interval), para se obter a distribuição a posteriori. Dessa forma, foram utilizadas distribuições a posteriori com 3.000 amostras. A dimensão de burn-in e intervalo de amostragem foram obtidos por análise prévia das cadeias geradas pelo amostrador de Gibbs utilizando o programa Gibanal (VAN KAAM, 1998), além de monitoração da convergência.

O erro de Monte Carlo foi obtido mediante o cálculo da variância das amostras retiradas para cada componente dividida pelo número de amostras. Assim, a raiz quadrada desse valor se refere à aproximação do desvio-padrão do erro associado ao tamanho da cadeia de Gibbs (VAN TASSELL; VAN VLECK, 1995). 


\section{RESULTADOS E DISCUSSÃO}

Os valores médios obtidos para idade ao primeiro parto (IPP), intervalo de partos (IP) e produção de leite (PL) foram 581,68 \pm 79,44; 322,89 \pm 132,02 dias e 226,99 \pm 89,72 $\mathrm{kg}$ de leite, respectivamente. Médias superiores a estas, foram obtidos por Lôbo e Silva (2005), avaliando caprinos das raças Saanen e Anglonubiana. Gonçalves et al. (2008), observaram médias de 365 dias para IPP e IP e média de $708 \mathrm{~kg}$ para a PL em cabras da raça Saanen. Torres-Vázquez et al. (2009), observaram valores idades ao primeiro parto em torno de 490 dias em animais da raça Saanen criados no México.

O valor médio da idade ao primeiro parto pode ser considerado elevado (581,68 $\pm 79,44$ dias), em torno de 19 meses. Possivelmente a IPP tenha sido influenciada pelo manejo da primeira cobertura, pois a identificação do cio era realizada somente duas vezes ao dia e apenas sob o aspecto visual. Gonçalves $e t$ al. (1997a e 1997b) relataram que, para conseguir resultados econômicos razoáveis, as cabritas devem alcançar o primeiro parto com 12 meses, independente do fotoperiodismo, porém, para criação intensiva no sudeste do país.

Todos os efeitos descritos no modelo foram significativos $(\mathrm{P}<0,05)$, com exceção do tipo de nascimento para IP e PL. A influência dos fatores ano e estação de parição explica-se como decorrente de flutuações do clima, especificamente a variação de pluviosidade entre e dentro dos anos estudados, que causam variações no desempenho em decorrência da estacionalidade na disponibilidade de alimentos, em termos qualitativos e quantitativos, mesmo com rebanho sendo submetido a manejo alimentar que visa contornar problemas de entressafra. Resultados similares aos obtidos nesta pesquisa foram relatados por Sarmento et al., (2003), Pimenta Filho et al., (2004), Torres-Vázquez et al. (2009) e Montaldo et al. (2010), que afirmaram ocorrer forte influência desses fatores em características reprodutivas e produtivas.

A não significância do tipo de nascimento sobre as características IP e PL pode ser atribuída ao manejo dos cabritos, que são aleitados artificialmente. Ou seja, a cabra ao receber estímulo de um cabrito é diferente do estímulo de dois ou três, onde, teoricamente, aumentaria a demanda de nutrientes por parte da gestação e para a síntese do leite, de modo a garantir o nascimento e sobrevivência das crias. Com isso, haveria aumento do período de serviço e, consequentemente, do intervalo de partos, pelo fato de a cabra necessitar de maior período de tempo para se recuperar e retornar à atividade reprodutiva. Como as cabras passam a receber estímulo único depois da parição, o do ordenhador, esse efeito seria anulado, corroborando com os relatos de Sarmento et al. (2003).

No que se refere a estimação de componentes de variância, observou-se que o desvio-padrão do erro de Monte Carlo foi pequeno e praticamente nulo, confirmando que o tamanho da cadeia amostral utilizada foi suficiente para obtenção de estimativas a posteriori das distribuições marginais dos componentes de (co)variância, ou seja, a convergência foi alcançada pela cadeia de Gibbs (Tabela 2).

Com relação ao erro de Monte Carlo, Van Tassel e Van Vleck (1995) afirmam que este erro é considerado pequeno quando seu valor somado à estimativa média da distribuição a posteriori dos coeficientes de herdabilidade não altera o valor desta estimativa, considerando até a segunda casa decimal. 
Além disso, pela análise gráfica da convergência observou-se a estabilização do processo iterativo (Figura 1). Dessa maneira, pode-se acreditar que as estimativas dos parâmetros são as médias das distribuições a posteriori, pois a condição de distribuição estacionária foi atingida.

As estimativas dos componentes de variância e parâmetros genéticos obtidos pela amostragem de Gibbs também permitiu a obtenção imediata de intervalos de credibilidade (Tabela 2), levando em conta a incerteza existente nos parâmetros simultaneamente, sendo, portanto, mais um indicador de precisão do método Bayesiano (Gelman et al., 1997).

A partir das estimativas dos componentes de (co)variância das características estudadas (Tabela 2 e 3), estimou-se os parâmetros genéticos, herdabilidade, repetibilidade e correlações genéticas pelos modelos uni e multicaracterísticas.

Tabela 2 - Distribuições a posterioiri dos componentes de (co)variância e parâmetros genéticos em análise unicaracterística

\begin{tabular}{|c|c|c|c|c|c|c|c|}
\hline \multirow{2}{*}{ Características } & \multirow{2}{*}{$\begin{array}{l}\text { Componentes } \\
\text { e Parâmetros }\end{array}$} & \multirow{2}{*}{ Média } & \multirow{2}{*}{ Moda } & \multirow{2}{*}{ Mediana } & \multicolumn{2}{|c|}{ IC-95\% } & \multirow{2}{*}{ EMC* } \\
\hline & & & & & LCL & UCL & \\
\hline \multirow{3}{*}{ IPP } & $\sigma_{a}{ }^{2}$ & 2014,19 & 1994,19 & 1921,76 & 1978,84 & 2049,55 & 0,01 \\
\hline & $\sigma_{e}^{2}$ & 12559,96 & 12659,96 & 12456,85 & 12484,51 & 12635,40 & 0,10 \\
\hline & $h^{2}$ & 0,14 & 0,13 & 0,13 & 0,13 & 0,14 & - \\
\hline \multirow{5}{*}{ IP } & $\sigma_{a}{ }^{2}$ & 795,73 & 801,38 & 779,12 & 772,88 & 808,58 & 0,0030 \\
\hline & $\sigma_{c}{ }^{2}$ & 652,49 & 772,11 & 615,34 & 639,06 & 776,91 & 0,0004 \\
\hline & $\sigma_{e}^{2}$ & 14222,86 & 14201,12 & 14154,29 & 14181,69 & 14264,04 & 0,2000 \\
\hline & $h^{2}$ & 0,05 & 0,05 & 0,05 & 0,04 & 0,05 & - \\
\hline & $t$ & 0,09 & 0,09 & 0,08 & 0,09 & 0,10 & - \\
\hline \multirow{5}{*}{ PL } & $\sigma_{a}^{2}$ & 729,42 & 719,61 & 720,04 & 717,60 & 767,24 & 0,0030 \\
\hline & $\sigma_{c}{ }^{2}$ & 641,11 & 629,82 & 648,55 & 629,80 & 652,43 & 0,0002 \\
\hline & $\sigma_{e}^{2}$ & 5524,09 & 5531,09 & 5498,42 & 5501,09 & 5547,09 & 0,0900 \\
\hline & $h^{2}$ & 0,11 & 0,10 & 0,10 & 0,10 & 0,11 & - \\
\hline & $t$ & 0,20 & 0,19 & 0,19 & 0,19 & 0,20 & - \\
\hline
\end{tabular}

IPP - idade ao primeiro parto; IP - intervalo de partos; PL - produção de leite; $\sigma_{a}{ }^{2}$ - variância devido os efeitos genéticos aditivos direto; $\sigma_{c}{ }^{2}$ - variância devido os efeitos de ambiente permanente (não correlacionados); $\sigma_{e}{ }^{2}$ variância residual; $h^{2}$ - herdabilidade da característica; $t$ - repetibilidade da característica; IC - intervalo de credibilidade a 95\%; LCL - Limite de credibilidade inferior; UCL - Limite de credibilidade superior; EMC* - erro de Monte Carlo com base na estimativa média das variâncias. 
Figura 1 - Processo da amostragem de Gibbs da variância genética aditiva (a) e variância residual (b) da característica IPP e variância aditiva (c e f), variância de ambiente permanente (d e g) e variância residual $(e$ e h) das características IP e PL, respectivamente, em função do número de interações da distribuição a posteriori.

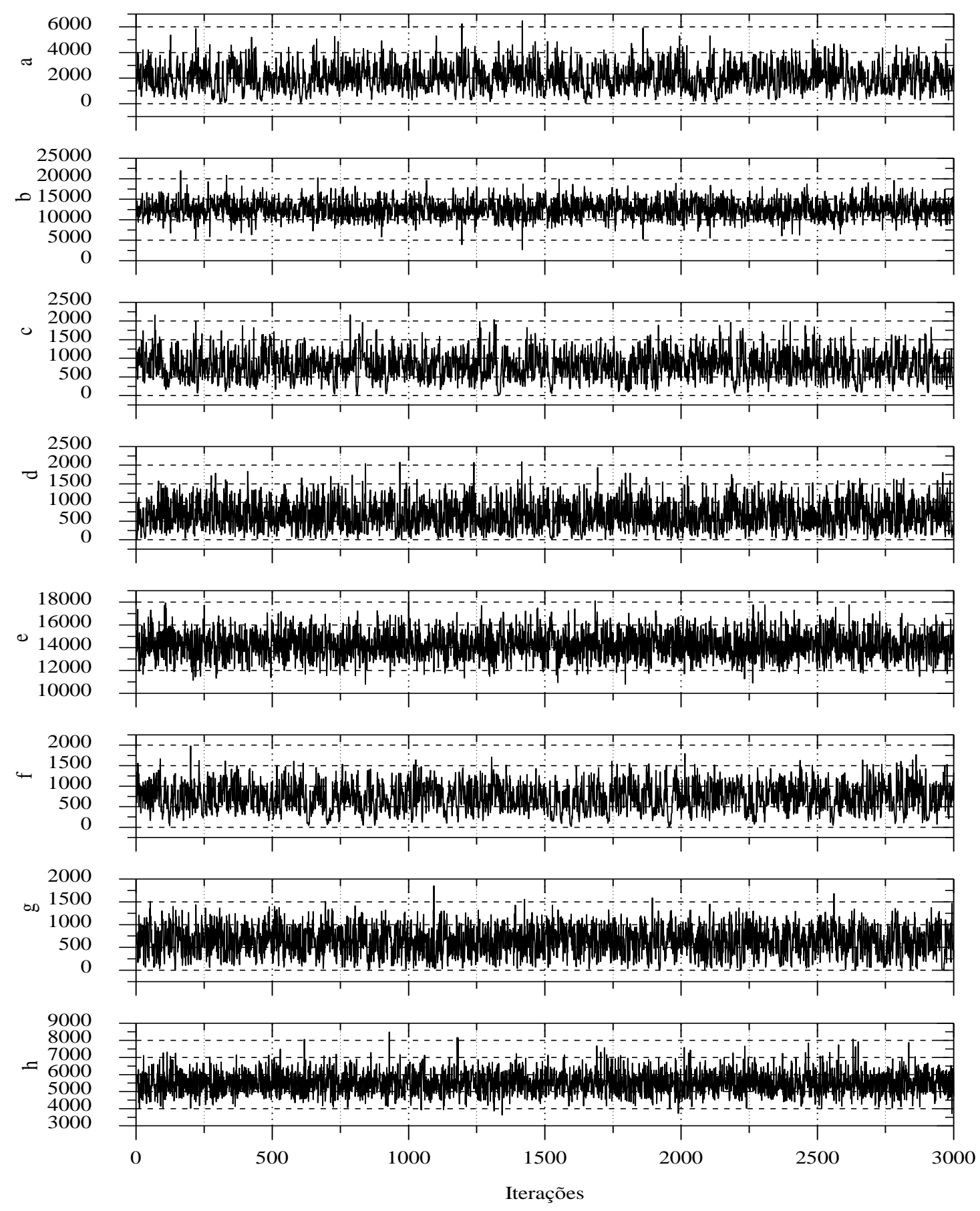


Tabela 3 - Médias a posteriori para os componentes de (co)variância e parâmetros genéticos em análise multicaracterística

\begin{tabular}{|c|c|c|c|c|c|c|c|c|c|c|c|c|c|c|}
\hline \multirow{3}{*}{ Características } & \multicolumn{13}{|c|}{ Componentes de (co)variância e parâmetros genéticos } & \multirow{3}{*}{ EMC } \\
\hline & \multirow{2}{*}{$\sigma_{a}^{2}$} & \multirow[b]{2}{*}{$\sigma_{a x, y}$} & \multirow{2}{*}{$\sigma_{c}^{2}$} & \multirow{2}{*}{$\sigma_{c x, y}$} & \multirow{2}{*}{$\sigma_{e}^{2}$} & \multirow[b]{2}{*}{$\sigma_{e x, y}$} & \multirow{2}{*}{$h^{2}$} & \multicolumn{2}{|c|}{ IC-95\% } & \multirow{2}{*}{$-r_{x, y}$} & \multirow{2}{*}{$t$} & \multicolumn{2}{|c|}{ IC-95\% } & \\
\hline & & & & & & & & LCL & UCL & & & LCL & UCL & \\
\hline IPP & 2365,89 & \multirow{2}{*}{788,92} & $\begin{array}{lll}- & \\
\end{array}$ & \multirow{2}{*}{ - } & 12450,82 & \multirow{2}{*}{$-1771,05$} & 0,16 & 0,15 & 0,17 & \multirow{2}{*}{0,52} & - & - & - & 0,001 \\
\hline IP & 965,39 & & 653,39 & & 14170,63 & & 0,06 & 0,04 & 0,06 & & 0,10 & 0,09 & 0,11 & 0,018 \\
\hline IPP & 2420,34 & \multirow{2}{*}{$-231,64$} & & \multirow{2}{*}{ - } & 12335,48 & \multirow{2}{*}{479,1} & 0,16 & 0,15 & 0,17 & \multirow{2}{*}{$-0,18$} & & - & - & 0,003 \\
\hline PL & 737,76 & & 649,95 & & 5522,64 & & 0,11 & 0,10 & 0,12 & & 0,20 & 0,18 & 0,21 & 0,022 \\
\hline IP & 756,09 & \multirow{2}{*}{98,75} & 455,46 & \multirow{2}{*}{$-251,54$} & 14384,94 & \multirow{2}{*}{4243,83} & 0,05 & 0,04 & 0,06 & \multirow{2}{*}{$-0,22$} & 0,08 & 0,07 & 0,11 & 0,029 \\
\hline PL & 799,83 & & 1163,75 & & 5385 & & 0,11 & 0,09 & 0,12 & & 0,27 & 0,23 & 0,28 & 0,006 \\
\hline
\end{tabular}

IPP - idade ao primeiro parto; IP - intervalo de partos; PL - produção de leite; $\sigma_{a}{ }^{2}$ - variância devido os efeitos genéticos aditivos direto; $\sigma_{a x, y}$ - covariâncigenética entre a primeira e segunda característica de cada análise; $\sigma_{c}{ }^{2}$ - variância devido os efeitos de ambiente permanente (não correlacionados); $\sigma_{c x, y}-$ covariância de ambiente permanente entre a primeira e segunda característica de cada análise; $\sigma_{e}{ }^{2}$ - variância residual; $\sigma_{e x, y}$ - covariância residual entre a primeira e segunda característica de cada análise; $h^{2}$ - herdabilidade da característica; $r_{x, y}$ - correlação genética entre a primeira e segunda característica de cada análise; $t$ repetibilidade da característica. IC - intervalo de credibilidade a 95\%; LCL - Limite de credibilidade inferior; UCL - Limite de credibilidade superior; EMC - erro de
Monte
Carlo
em
função
estimativa
da
variação
da
herdabilidade
média. 
As estimativas de variância genética foram abaixo das relatadas na literatura (SARMENTO et al., 2003; PIMENTA FILHO et al., 2004; LÔBO; SILVA, 2005), exceto para IPP. A estimativa destes componentes mostra-se importante para o conhecimento da estrutura da população e o delineamento de estratégias de seleção adequadas, inclusive para utilização na estimativa de índices de seleção.

Destaca-se que as estimativas de variância genética aditiva nas análises multicaracterística foram superiores às estimadas em análises unicaracterísticas, principalmente para IPP e IP. Esse aumento na diferença do componente de variância genético aditivo no modelo reflete a remoção do vício decorrente da seleção a maior número de informações além das relações entre as características, o que possibilitaram resgatar parte da variância aditiva direta, levando a estimativas maiores de herdabilidade (SARMENTO et al., 2006).

Mesmo com o aumento no valor da estimativa de variância genética aditiva na análise multicaracterística, com redução expressiva na variância residual, a variância fenotípica estimada foi a praticamente a mesma nas duas análises. Dessa forma, o aumento do número de informações nas análises conjuntas utilizando as relações entre os animais nos diferentes níveis de variabilidade permitiram filtrar maiores proporções de variância genética aditiva, que antes eram adicionadas ao componente residual. Ou seja, a utilização de componentes de covariância entre as variáveis possibilitou a obtenção de estimativas de variância mais apuradas, diminuindo o componente da variância residual a favor dos componentes de variância genéticos aditivos.

A decisão quanto a utilizar modelos multicaracterísticas, ao invés de unicaracterística, irá depender, principalmente, das herdabilidades e correlações entre as características, pois conforme a combinação entre tais parâmetros, a eficiência entre esses dois modelos pode ser semelhante (BALDI et al., 2010).

Em análise unicaracterística obtiveram-se valores de herdabilidade de 0,14; 0,05 e 0,10 para IPP, IP e PL, respectivamente. Na mesma ordem, para análise bicaracterística, as herdabilidades foram de 0,16 ; 0,06 e 0,11. Dessa forma, é fácil observar que houve sensível melhora nas estimativas e incremento nos componentes de variância. $\mathrm{O}$ aumento da estimativa de herdabilidade com modelos de multicaracterísticas também foi relatado por Sarmento et al. (2006).

Essas estimativas de herdabilidade indicam relação baixa entre o fenótipo e genótipo dos indivíduos. Portanto, a seleção de fêmeas pelo fenótipo promoverá lento incremento genético na população. Assim, a partir dos resultados obtidos e pelo fato de as características serem limitadas ao sexo, para os reprodutores, o teste de progênie é o método indicado como o mais eficaz para seleção de bodes destinados à reprodução vislumbrando o incremento da IPP, IP e PL.

A estimativa da herdabilidade para o IP e PL mostram que o fenótipo não é um bom indicador do genótipo dos indivíduos. Resultados similares foram, obtidos por Bagnicka et al. (2007). Assim, espera-se que a seleção não proporcione ganhos genéticos satisfatórios. Dessa forma, Mugambi et al. (2007) e Barbosa Neto et al. (2010) recomendam a utilização de cruzamentos, mantendo elevado nível de heterozigose, o que contribuiria para um melhor desempenho de forma mais rápida. Como as 
herdabilidades estimadas para o IP foram baixas, deve-se ressaltar que a expressão desta característica pode está intimamente ligada a PL, o que pode comprometer a avaliação do verdadeiro potencial reprodutivo dos animais, uma vez que os caprinocultores tendem a aumentar o IP por estenderem a lactação dos animais.

Lôbo e Silva (2005) obtiveram herdabilidades de 0,49 e 0,21; 0,01 e 0,06 e 0,10 e 0,12 para IPP, IP e PL com as raças Anglonubiana e Saanen, respectivamente. Na literatura é possível observar variações nas estimativas para os parâmetros genéticos em estudo, possivelmente pela pouca informação utilizada nas análises. Vale ressaltar que a estimativa desses parâmetros foram realizadas pelo método da máxima verossimilhança restrita (REML). Garcia-Peniche et al. (2012) estudando algumas raças caprinas, obtiveram herdabilidades variando entre 0,16 a 0,41 para idade ao primeiro parto; 0,02 a 0,21 para intervalo de partos; e de 0,36 e 0,61 para produção de leite. Fica evidente que a herdabilidade varia entre raças, famílias, populações e pode ser influenciada pelo ambiente.

A correlação genética estimada entre IPP e IP foi positiva e moderada. Este fato pode ser em virtude da criação voltar-se à produção de leite, pois animais precoces tendem a ser reprodutivamente superiores. Desse fato, resulta que ao apresentarem primeiro parto precocemente, estes animais tenderão a apresentar menores intervalos de parto. No entanto, sabe-se que a resposta correlacionada não depende exclusivamente da correlação genética entre as características, mas também das herdabilidades das características envolvidas no processo de seleção. Dessa forma, apesar da moderada correlação genética entre as características IPP e IP, a resposta correlacionada pela seleção indireta, mesmo sendo eficiente, poderá resultar em ganhos genéticos baixos. Montaldo et al. (2010) relatam resultados similares aos desta pesquisa para a raça Saanen, utilizando modelo multicaracterística, além disso, em todos as análises a característica intervalo de parto apresentou herdabilidade baixa $(0,02-0,15)$, corroborando com os resultados obtidos.

As estimativas de repetibilidade para as características IP e PL apresentaram-se, de forma geral, com baixa magnitude (Tabela 3), de modo que serão necessárias mais mensurações nos animais para representar sua real capacidade de produção, ou seja, uma única observação feita no indivíduo apresenta pequeno valor para a sua seleção, havendo necessidade de que novas ocorrências sejam consideradas. Destaca-se que com o aumento de mensurações nos animais pode-se minimizar os possíveis erros decorrentes da coleta dos dados. Estimativas de repetibilidade inferiores para estas características foram relatadas por Lôbo e Silva (2005) e superiores foram registradas por Pimenta Filho et al. (2004) e GarciaPeniche et al. (2012).

As características que se repetem ao longo da vida do animal, a exemplo do IP e da PL, se expressam em diferentes intensidades em cada ocorrência, e o coeficiente de repetibilidade estimado de 0,10 e 0,20 , respectivamente, representam a correlação entre os desempenhos sucessivos dos animais. Apesar de baixo, este coeficiente mostra-se como importante ferramenta quando se trabalha com animais que apresentam várias medidas da mesma característica, o que pode auxiliar a predição de desempenhos futuros dos animais. 


\section{CONCLUSÃO}

A seleção para menor idade ao primeiro parto favorece a eficiência reprodutiva com a diminuição do intervalo de partos. A precocidade ao primeiro parto interfere na expressão do potencial produtivo com a diminuição da produção de leite. Análises multicaracterísticas possibilitaram resgatar parte da variância aditiva direta, levando a obtenção de estimativas de herdabilidade superiores que às obtidas em análise unicaracterística.

\section{REFERÊNCIAS}

BAGNICKA, E. et al. Heritability for reproduction traits in Polish and Norwegian populations of dairy goat. Small Ruminant Research, v. 68, p. 256-262, 2007.

BALDI, F.; ALENCAR, M.M.; ALBUQUERQUE, L.G. Estimativas de parâmetros genéticos para características de crescimento em bovinos da raça Canchin utilizando modelos de dimensão finita. Revista Brasileira de Zootecnia, v. 39, n. 11, p. 2409-2417, 2010.

BARBOSA NETO, A.C.; et al. Efeitos aditivos e não-aditivos em características de crescimento, reprodutivas e habilidade materna em ovinos das raças Santa Inês, Somalis Brasileira, Doper e Poll Dorset. Revista Brasileira de Zootecnia, v. 39, n. 9, p. 1943-1951, 2010.

CARNEIRO JÚNIOR, J.M.et al. Influência da informação a priori na avaliação genética animal utilizando dados simulados. Revista Brasileira de Zootecnia, v. 34, n. 6, p. 1905-1913, 2005.

FALCÃO, A.J.S. et al.. Efeitos do número de animais na matriz de parentesco sobre estimativas de componentes de variância para produção de leite usando os métodos de Máxima Verossimilhança Restrita e Bayesiano. Revista Brasileira de Zootecnia, v. 38, n. 8, p. 1478-1487, 2009.

FARIA, C.U. et al. Inferência Bayesiana e sua aplicação na avaliação genética de bovinos da raça Nelore: revisão bibliográfica. Ciência Animal Brasileira, v. 8, p. 75-86, 2007.

GARCIA-PENICHE, T.B. et al. Breed differences over time and heritability estimates for production and reproduction traits os dairy goats in the United States, Journal of Dairy Science, v. 95, p. 2707-2717, 2012.

GELMAN, A. et al. Bayesian data analysis. London: Chapman Hall, 1997. 526p.

GONÇALVES, A.L.; LANA, R.P.; VIEIRA, R.A.M. Avaliação se sistemas de produção de caprinos leiteiros na região sudeste do Brasil. Revista Brasileira de Zootecnia, v. 37, n. 2, p. 366-376, 2008.

GONÇALVES, H.C. et al. Fatores genéticos e de meio no intervalo de partos de caprinos leiteiros.

Revista Brasileira de Zootecnia, v.26, n.5, p. 905-913, 1997a.

GONÇALVES, H.C. et al. Fatores genéticos e de meio na idade ao primeiro parto de caprinos leiteiros. Revista Brasileira de Zootecnia, v.26, n.3, p. 485-493, 1997b.

LASSEN, J.et al. An approximate multitrait model for genetic evaluation in dairy cattle with a robust estimation of genetic trends. Genetics Selection Evolution. v. 39, p 353-367, 2007. 
LÔBO, R.N.B.; SILVA, F.L.R. Parâmetros genéticos para características de interesse econômico em cabras da raça Saanen e Anglo-nubiana. Revista Ciência Agronômica, v. 36, n. 1, p. 104-110, 2005.

MONTALDO, H.H. et al. Short communication: genetic and evironmental relationships between Milk yield and kidding interval in dairy goatas. Journal of Dairy Science, v. 93, p. 370-372, 2010.

MUGAMBI, J.N.; WAKHUNGU, J.W.; INYANGALA, B.O. Evaluation of the performance of the Kenya goat composites: additive and non-additive genetic parameters. Small Ruminant Research, v.72, p.149156, 2007.

PIMENTA FILHO, E.C.; SARMENTO, J.L.R.; RIBEIRO, M.N. Efeitos genéticos e ambientais que afetam a produção de leite e duração da lactação de cabras mestiças no estado da Paraíba. Revista Brasileira de Zootecnia, v. 33, n. 6, p. 1426-1431, 2004.

SARMENTO, J.L.R. et al. Fatores genéticos e de ambiente sobre o intervalo de partos em cabras leiteiras no semi-árido Nordestino. Revista Brasileira de Zootecnia, v. 32, n. 4, p. 875-879, 2003.

SARMENTO, J.L.R.; et al. Estimação de parâmetros genéticos para características de crescimento de ovinos Santa Inês utilizando modelos uni e multicaracterísticas. Arquivo Brasileiro de Medicina Veterinária e Zootecnia, v. 58, n. 4, p. 581-589, 2006.

TORRES-VÁZQUEZ, J.A.et al. Genetic and phenotypic parameters of Milk yield, Milk composition and age at first kidding in Saanen goats from Mexico. Livestock Science, v. 126, p. 147-153, 2009.

VAN KAAM, J.B.C.H.M. Gibanal 2.9: analyzing program for Markov Chain Monte Carlo sequences. Wageningen, The Netherlands: Department of Animal Science, Wageningen Agricultural University, 1998.

VAN TASSELL, C. P., VAN VLECK, L. D. A manual for use of MTGSAM: A set of FORTRAN programs to apply Gibbs sampling to animal models for variance component estimation. Lincoln: USDA, ARS, 1995. 